\title{
Calibration of an Electrical Analog Model of Liver Hemodynamics in Fontan Patients
}

\section{Elyar Abbasi Bavil}

Department of Mechanical and Industrial Engineering, University of Toronto, Toronto, Canada

5 King's College Road, Toronto, ON M5S 3G8

e-mail: elyar.abbasibavil@mail.utoronto.ca

\section{Matthew G. Doyle}

Department of Mechanical and Industrial Engineering,

Division of Vascular Surgery, Peter Munk Cardiac Centre, University Health Network, Department of Surgery, University of Toronto, Toronto, Canada

5 King's College Road, Toronto, ON M5S 3G8

e-mail: mg.doyle@utoronto.ca

\section{Charlotte Debbaut}

IBiTech-bioMMeda, Department of Electronics and Information Systems, Ghent University, Ghent, Belgium

Campus UZ - Blok B - entrance 36, Corneel Heymanslaan 10, 9000 Gent, Belgium

e-mail: charlotte.debbaut@UGent.be

\section{Rachel M. Wald}

Division of Cardiology, Peter Munk Cardiac Centre, University Health Network, University of Toronto, Toronto, Canada

5N 517, 585 University Ave, Toronto, ON M5G 2N2

e-mail: rachel.wald@uhn.ca

\section{Luc Mertens}

Division of Cardiology, Peter Munk Cardiac Centre, University Health Network, Division of Cardiology, Department of Pediatrics, The Labatt Family Heart Centre, The Hospital for Sick Children, University of Toronto, Toronto, Canada

555 University Ave, Toronto, ON M5G 1X8

e-mail: luc.mertens@sickkids.ca

\section{Thomas L. Forbes}

Division of Vascular Surgery, Peter Munk Cardiac Centre, University Health Network, Department of Surgery, University of Toronto, Toronto, Canada

585 University Ave, Toronto, ON M5G 2N2

e-mail: thomas.forbes@uhn.ca

\section{Cristina H. Amon (Corresponding Author)}

Department of Mechanical and Industrial Engineering,

Institute of Biomedical Engineering, University of Toronto, Toronto, Canada 
Journal of Biomechanical Engineering

5 King's College Road, Toronto, ON M5S 3G8

e-mail: cristina.amon@utoronto.ca

Phone: 416-978-2272

Fax: 416-978-7753 


\begin{abstract}
Fontan associated liver disease is a common complication in patients with Fontan circulation, who were born with a single functioning heart ventricle. The hepatic venous pressure gradient (HVPG) is used to assess liver health and is a surrogate measure of the pressure gradient across the entire liver (portal pressure gradient). However, it is thought to be inaccurate in Fontan patients. The main objectives of this study were (1) to apply an existing detailed lumped parameter model of the liver to Fontan patients using patientspecific clinical data and (2) to determine whether HVPG is a suitable measurement of portal pressure gradients in these patients.

An existing lumped parameter model of the liver blood circulation was applied and tuned to simulate patient-specific liver hemodynamics. Geometries were collected from seven adult Fontan patients and used to evaluate model parameters. The model was solved and tuned using waveform measurements of flows, inlet and outlet pressures.

The predicted ratio of portal to hepatic venous pressures is comparable to in vivo measurements. The results confirmed that HVPG is not suitable for Fontan patients, as it would underestimate the portal pressures gradient by a factor of 3 to 4 .

Our patient-specific liver model provides an estimate of the pressure drop across the liver, which differs from the clinically used metric HVPG. This work represents a first step towards models suitable to assess liver health in Fontan patients and improve its long-term management.
\end{abstract}

\title{
Key Words
}

Fontan procedure, biological system modelling, electrical analog model, liver hemodynamics, fluid dynamics. 


\section{INTRODUCTION}

Fontan associated liver disease is increasingly being recognized in patients who have undergone the Fontan palliation where the systemic venous return is directed to the pulmonary arteries in the absence of an interposed pumping chamber due to underlying single ventricle physiology [1]. Exposure of the liver to elevated systemic venous pressures shortly after Fontan completion leads to congestion [2]. Chronic liver congestion can result in morphologic changes, including generalized fibrosis and cirrhotic remodeling, which may result in an increased risk of hepatocellular carcinoma [3]. Liver disease and hemodynamics are tightly linked in these patients, as evident by the reduction of flow rates in the portal vein (PV) (inlet) and hepatic vein (HV) (outlet) in patients with morphologic evidence of liver disease [4].

An important hemodynamic parameter, the hepatic venous pressure gradient (HVPG), is a widely used clinical tool for identifying the degree of liver fibrosis and elevated pressure in the PV system. It is calculated as the difference between the pressures measured at the HV outlet and the sinusoids (represented by the wedged HV pressure), where the sinusoids are liver-specific capillaries [5],[6]. Studies have shown that in many liver conditions the wedged $\mathrm{HV}$ pressure is a reasonable surrogate for the PV pressure, which cannot easily be measured directly using a pressure catheter. Hence, the HVPG would approximate the pressure gradient across the liver (between the PV and HV), referred to as the portal pressure gradient (PPG) [8],[9]. Large HVPG values (> $10 \mathrm{mmHg}$ ) have been associated with severe liver fibrosis and clinically significant portal hypertension [6]. In Fontan patients however, high venous pressures are retrogradely transmitted to the hepatic veins 
(so-called "hepatic afterload") and in turn to the sinusoids. This results in dilation of the sinusoids which reduces resistance and diminishes the HVPG to approximately $1-2 \mathrm{mmHg}$ [8],[9]. Despite consistently low HVPGs, a case study by Velpula et al. detailing the results of catheter measurements in a Fontan patient with liver disease reported a PPG value of 9 mmHg and a HVPG measurement of $3 \mathrm{mmHg}$, suggesting that HVPG may not be a good surrogate for PPG or indicator of liver fibrosis in Fontan patients [10].

Because PPG cannot easily be measured clinically, computational models, based on patient-specific clinical data, can be used to estimate PPG and test the hypothesis that HVPG is not a good approximation for PPG in Fontan patients. One such type of computational models is lumped parameter models (LPMs), which are computationally inexpensive models to simulate fluid flow through a network based on analogies between electrical circuits and fluid mechanics. These models can focus on a specific organ, such as the liver, or examine the entire circulatory system. In the context of Fontan patients, many LPMs have been developed with different degrees of complexity, often simulating the cardiac hemodynamics using higher-level models of the whole systemic circulation [11]-[14]. However, a detailed LPM of the liver has yet to be developed for Fontan patients to better understand their unique liver hemodynamics.

Several computational fluid dynamics models of liver hemodynamics have been developed based on single liver samples [15]-[17]. These models are computationally heavy and not suitable for routine patients-specific modelling. LPMs have been developed for the liver, most with low to moderate degrees of complexity [18]-[21]. Wang et al. [21] developed 
an LPM of the liver coupled to a 1-D model of the systemic circulation to analyze the difference between HVPG and PPG in different pathological cases of portal hypertension. Audebert et al. [20] developed an LPM of the circulatory system, including the hepatic circulation, to explore the effect of changing vascular resistances on the hepatic and global circulation hemodynamics during cirrhogenesis in rats. Van Der Plaats et al. [19] developed a detailed model of a canine liver, which included information on the inner vessel generations. Debbaut et al. [18] used the same concept to develop a model of the human liver, based on a liver that was discarded for transplantation after failed reallocation. This liver underwent vascular corrosion casting and micro-CT imaging. Detailed image processing and analysis allowed for estimation of the internal vascular topology and geometries as input for the model.

Previous LPMs of liver hemodynamics were not based on in vivo patient-specific measurements, nor were they applied to livers of Fontan patients. The aims of the current study are therefore to (i) apply an existing liver LPM by Debbaut et al. [18] to Fontan patients using patient-specific clinical data and (ii) use the tuned models to provide PPG estimates in these patients to test the hypothesis that HVPG is not a reasonable estimate of PPG in Fontan patients.

\section{METHODS}

\section{Study Population}

This was a single-center retrospective cohort study of Fontan patients seen in the adult congenital cardiac clinic at Toronto General Hospital between December 2014 and July 
2016. The patients underwent phase-contrast cardiac magnetic resonance imaging (PCCMR) with inclusion of imaging of the abdominal vessels. Our inclusion criterion was the availability of a cardiac catheter procedure within 2 years from their PC-CMR that included pressure waveform readings. The study was approved by the institutional research ethics board (REB 10-0398-BE) and patient consent was waived.

We screened a total of 30 patients for this study and 7 patients were selected who met the inclusion criteria (Table 1). Based on an imaging-based assessment of their livers, the population was divided into two groups using a previously described method [4]: group I with morphological evidence of liver disease (patients 1-4) and group II without (patients 5-7). The average age at the time of PC-CMR was $34.1 \pm 8.3$ years (group I: $34.0 \pm 9.6$ years, group II: $34.3 \pm 8.1$ years). The population is representative of the two main types of Fontan connections with 4 patients having atriopulmonary (AP) connections, where the right atrium is connected to the right pulmonary artery, and 3 patients having the total cavopulmonary connection (TCPC) (2 lateral tunnel variants and 1 extracardiac variant), where the inferior and superior vena cavae (IVC \& SVC) are directly connected to the pulmonary arteries [22]. Patient 1 experienced the most severe liver disease and died a year after the PC-CMR due to complications of hepatocellular carcinoma.

\section{Magnetic Resonance Imaging Acquisition}

All PC-CMR scans were performed on a 1.5 Tesla scanner (Magnetom Avanto Fit; Siemens Healthcare, Erlangen, Germany). For evaluation of cardiac and abdominal flows, fast cine PC analysis was performed during free breathing. Typically, 30 images (256x166 
pixels, slice thickness of $4 \mathrm{~mm}$, and in-plane spatial resolution of $1.3 \times 1.3 \mathrm{~mm}$ ) per cardiac cycle were acquired and an encoded velocity was selected based on the vessel of interest. PC-CMR images were analyzed using QFlow (Medis Medical Imaging Systems, Leiden, the Netherlands) to calculate mean flow rates (averaged over the blood vessel crosssection) and extract flow waveforms over the cardiac cycle. The region of interest was detected automatically using the contour generation option by applying appropriate inflation or deformation values. The flow rates were calculated without applying background subtraction.

\section{Geometric and Hemodynamic Data Collection}

As shown in Figure 1, the liver has two inlets, the hepatic artery (HA), which delivers oxygenated blood, and the PV, which delivers nutrient rich, partially deoxygenated blood, and three outlets, the left, middle, and right HVs [23]. Blood from the HA and PV trees mixes in the sinusoids and then travels through the HV tree before exiting the liver through the HVs into the IVC.

The geometric parameters (radii, lengths, and number of vessels per generation) for all visible liver blood vessels were measured from PC-CMR scans. Typically, only the first 2 or 3 vessel generations were visible with sufficient resolution to enable accurate measurements. Specifically, we were able to measure geometric parameters for 3 generations for patients 4,5 and 7, and 2 generations for all other patients. The geometric parameters of the remaining generations were approximated from measurements by Debbaut et al. [18] as described below. Using Qflow, radii were calculated from the vessel 
cross-sectional areas assuming circular cross-sections. For each vessel, radii were measured at three cross-sections along the vessel and averaged. Similarly, Qflow was used to determine vessel lengths by measuring the centerline distance along each vessel. Using the approach proposed by Debbaut et al. [18], vessels were assigned to a particular generation based on the branching patterns associated with bifurcations, trifurcations, and side branches. The HA, PV, and HV were defined as generation 1. Subsequent vessels branching from generation $j$ in a bifurcating or trifurcating manner were defined as generation $j+1$. Side branches were assigned to the generation following the one they originated from, unless their radii were much smaller, in which case, they were assigned to the generation with comparable radii.

HA and PV flow waveforms were collected using their PC-CMR (Figure 1b). HV flow waveforms could not be measured and were solved for as a model output. HA pressure waveforms were not measured due to small vessel radii through which the pressure catheter could not be navigated. To estimate these pressures, we collected the ascending aorta pressure waveforms and the brachial cuff pressures at the time of measurement. HV pressure waveforms were collected, where available, and, in their absence, the pressure waveforms at the IVC above the HV confluence were used instead, because their values are comparable. We also collected the mean wedged HV pressure as an estimate of the pressure at the sinusoids. PV pressures were not measured due to complications with location accessibility and will be estimated as an output of our model. All flow and pressure signals were collected with a sampling frequency of $200 \mathrm{~Hz}$. Because flow and pressure waveforms were collected at different times, they had slightly different periods. To account 
for this, the periods of the pressure waveforms were scaled to match the periods of the corresponding flow waveforms.

\section{Lumped Parameter Model of the Liver}

Using patient-specific geometric and hemodynamic data, along with a few assumptions, an existing detailed LPM of the liver can be used to simulate personalized liver hemodynamics in Fontan patients. Figure 2 provides an outline of the steps taken to define and solve the LPM, which are described in more detail in the following sections.

We propose a liver LPM tailored to Fontan patients (Figure 3a) by applying the liver perfusion model of Debbaut et al. [18], which was originally developed for liver perfusion inside a hypothermic machine perfusion device. The model consists of three major compartments representing the PV, HA, and HV trees. Each compartment consists of several blood vessel generations, for which the total number of generations in the PV, HA, and HV trees are denoted by $m, n$, and $q$, respectively. The PV and HA trees merge at the level of the sinusoids, which is the last generation of the HV tree. Based on geometric measurements and extrapolations by Debbaut et al. [18], the values of $m, \mathrm{n}$ and $q$ are set to 16,18 , and 13 , respectively. Each generation is defined by an electric circuit referred to as a $\pi$-filter, which contains four electrical components: serial resistors $R_{s i j}$ representing the vascular resistance, inductors $L_{i j}$ representing the blood inertia, capacitors $C_{i j}$ representing the blood vessel elasticity, and parallel resistors $R_{p i j}$ representing the blood vessel viscoelasticity, where $i=P V, H A, H V$ and $j$ represents the generation number. The main difference between the model proposed by Debbaut et al. [18] and ours is the inlet boundary 
condition for the portal vein. We used an inlet flow boundary condition $\left(Q_{\mathrm{PV}}\right)$ instead of their inlet pressure boundary condition $\left(P_{\mathrm{PV}}\right)$ due to the availability of clinical data. We also used an inlet pressure boundary condition at the hepatic artery $\left(P_{\mathrm{HA}}\right)$, which is consistent with the model of Debbaut et al. [18]. The main model output parameters are the pressures at the $\mathrm{HV}$ outlet $\left(P_{\mathrm{HV}}\right)$ and the $\mathrm{PV}$ inlet $\left(P_{\mathrm{PV}}\right)$. The model assumes conservation of mass, that is $Q_{\mathrm{HA}}+Q_{\mathrm{PV}}=Q_{H V}$. The circuit is closed by a resistance $R$ at the HV end, which represents the downstream resistance of the distal circulatory system, estimated by $R=P_{\mathrm{HV}} / Q_{\mathrm{HV}}$.

\section{Governing Equations and Model Parameter Evaluation}

De Pater et al. [24] defined the following governing equations for the simplified NavierStokes equations for fluid flow through an elastic tube

$$
\begin{gathered}
-\frac{\partial P}{\partial z}=\frac{8 \mu}{\pi r^{4}} Q+\frac{4 \rho}{3 \pi r^{2}} \frac{\partial Q}{\partial t}, \\
-\frac{\partial Q}{\partial z}=\frac{3 \pi r^{3}}{2 E_{\text {Wall }} h_{\text {Wall }}} \frac{\partial P}{\partial t}+\frac{\mu_{\text {Wall }}}{E_{\text {Wall }}} \frac{\partial^{2} Q}{\partial z \partial t},
\end{gathered}
$$

where $z$ is the axial direction, $P$ is pressure $[\mathrm{Pa}], \mu$ is blood viscosity $[\mathrm{Pa} \cdot \mathrm{s}], r$ is the vessel radius $[\mathrm{m}], \rho$ is blood density $\left[\mathrm{kg} / \mathrm{m}^{3}\right], Q$ is flow rate $\left[\mathrm{m}^{3} / \mathrm{s}\right], E_{\text {wall }}$ is the vessel wall Young's modulus $[\mathrm{Pa}], h_{\text {wall }}$ is the vessel wall thickness $[\mathrm{m}], \mu_{\text {wall }}$ is the vessel wall viscoelasticity $[\mathrm{Pa} \cdot \mathrm{s}]$. 
Using analogies between fluid flow and electric circuits, these equations can be rewritten for a transmission line of length $\Delta \mathrm{z}$ (Figure 3c) in terms of the four electrical components $R_{s i j}, L_{i j}, C_{i j}$, and $R_{p i j}$ as

$$
\begin{gathered}
-\frac{\partial P}{\partial z}=R_{s i j} Q+L_{i j} \frac{\partial Q}{\partial t} \\
-\frac{\partial Q}{\partial z}=C_{i j} \frac{\partial P}{\partial t}+R_{p i j} C_{i j} \frac{\partial^{2} Q}{\partial z \partial t} .
\end{gathered}
$$

To model a blood vessel of finite length, $l$, the transmission line model was adapted to a $\pi$ filter circuit and the electrical parameters had to be multiplied by $l$. Finally, to account for the multiple vessels in each generation, the parameters $R_{s i j}, L_{i j}$ and $R_{p i j}$ were divided by and $C_{i j}$ was multiplied by the number of vessels in each generation $N$, leading to

$$
\begin{aligned}
& R_{s_{i j}}=\frac{8 \mu l}{\pi r^{4} N} \quad\left[\mathrm{~N} \cdot \mathrm{s} / \mathrm{m}^{5}\right] \\
& L_{i j}=\frac{1.33 \rho l}{\pi r^{2} N} \quad\left[\mathrm{~N} \cdot \mathrm{s}^{2} / \mathrm{m}^{5}\right] \\
& C_{i j}=\frac{l^{2} N}{2 c_{p}^{2} L} \quad\left[\mathrm{~m}^{5} / \mathrm{N}\right], \\
& R_{p_{i j}}=\frac{2 \times 10^{-6}}{C N} \quad\left[\mathrm{~N} \cdot \mathrm{s} / \mathrm{m}^{5}\right]
\end{aligned}
$$

where the constant $2 \times 10^{-6}$ is $h_{\text {wall }} / E_{\text {wall }}$ for human livers, as defined by Debbaut et al. [18], and $c_{p}$ is the pulse wave velocity $[\mathrm{m} / \mathrm{s}]$ defined as

$$
c_{P}=\sqrt{\frac{h_{\text {wall }}}{r} \frac{E_{\text {wall }}}{2 \rho}} \quad[\mathrm{m} / \mathrm{s}] .
$$


Blood flow in the liver was assumed to be laminar [19] and blood was assumed to behave as a Newtonian fluid with constant viscosity $\mu=3.5 \times 10^{-3} \mathrm{~Pa} \cdot \mathrm{s}$ [18], [25]. Blood density in the HA tree was assumed to be $\rho=1050 \mathrm{~kg} / \mathrm{m}^{3}$ and, based on Ref. [26], blood density in the PV tree was assumed to be $\rho=2000 \mathrm{~kg} / \mathrm{m}^{3}$. Blood density in the PV is almost twice as large as in the HA tree due to the nutrient rich blood coming from the intestines and the spleen. Blood in the sinusoids is modeled with a density calculated as a weighted average of the densities in the HA and PV trees based on their flow ratio. Blood in the rest of the HV tree was assumed to have a density of $\rho=1050 \mathrm{~kg} / \mathrm{m}^{3}$, because it is assumed that nutrients have been eliminated across the sinusoids.

Parameters $l, r$, and $N$ could only be measured accurately for generations 1-3 for patients 4,5 , and 7 , and generations 1-2 for all other patients based on the quality and number of slices available in PC-CMR images. For the remaining generations, we used the detailed measurements made by Debbaut et al. [18]. The impact of changing the radii (most sensitive parameter) of the inner generations on the model output is studied in a sensitivity analysis.

Because the parameters $h_{\text {wall }}$ and $E_{\text {wall }}$ could not be measured from MR images, the following assumptions were implemented for the calculation of $c_{\mathrm{p}}$ :

(1) For PV vessel wall thicknesses, the PV thickness and radius measurements for 36 pigs grouped into 6 age groups [27] were used to form the following linear correlation, with an $\mathrm{R}^{2}$ value of 0.9 , used to calculate $h_{P V}$

$$
h_{P V}=0.0829\left(r_{P V}\right)+0.0405[\mathrm{~mm}] .
$$


(2) HV and PV thicknesses have been shown to be comparable [28]. Consequently, Equation 10 was also used to calculate $\mathrm{HV}$ vessel wall thicknesses, $h_{H V}$.

(3) Based on Ref. [18], the Young's modulus for the PV inlet and HV outlet were assumed to be $E_{\text {wall }}=400 \mathrm{kPa}$, while for the last generation of the PV and $\mathrm{HV}$ trees (sinusoids), it was assumed to be $E_{\text {wall }}=2000 \mathrm{kPa}$. Linear interpolation was used for the generations in between.

(4) For the HA tree, in the absence of detailed information on vessel wall thicknesses needed to calculate $c_{p}$, we assumed $c_{p}=9.35 \mathrm{~m} / \mathrm{s}$ for the HA inlet based on a value proposed by Willemet et al. [29] for a typical human HA. For the rest of the HA tree, linear interpolation was used with the lower bound of $9.35 \mathrm{~m} / \mathrm{s}$ and the upper bound of $c_{p}$ calculated at the sinusoids.

Debbaut et al. [18] determined the sensitivity of their model to a two-fold variation in $c_{p}$ and found only a slight influence of $c_{p}$ on the amplitude and phase angle of the solution. Therefore, our assumptions for evaluating $c_{p}$ seem reasonable.

\section{Model Solution}

The model was solved in both steady state (direct current (DC)) and transient modes (alternating current (AC)) using the superposition theorem [18]. The superposition theorem is typically used to solve electrical networks with two or more sources. This theorem states that in a linear electrical circuit with several sources, the current and voltage over any element in the circuit is the sum of the responses (currents and voltages) produced by each source acting independently (where all the other sources are replaced by their internal impedances). The steady state solution (DC), used to calculate PPG values, entailed 
shorting out the inductors and open circuiting the capacitors in order to calibrate $R \mathrm{~s}$, which determines the mean pressure drop and flows in the liver. The transient solution (AC) involved working in the frequency domain based on the Fourier transform theory and is used for calibrating the rest of the parameters $\left(L, C, R_{p}\right)$ as well as evaluating the pressure and flow waveforms across the liver generations. To that end, the transient solution takes advantage of the superposition approach by alternating between the inlet sources. First, the PV current source is treated as open circuit, while the HA pressure source is active and the flow and pressure terms at each generation are evaluated. Secondly, the HA pressure source is shorted out, while the PV current source is active, and the same terms are evaluated again. The final transient solution adds up the results of both stages. An in-house code was written in MATLAB (MathWorks, Natick, MA) to solve the model and perform the calibration procedure.

\section{Model Calibration}

Our model implements patient-specific measurements of the vascular geometry for as many blood vessel generations as available, complemented by geometric data from Debbaut et al. [18] for the remaining inner vessel generations. Doing so, in combination with assumptions for evaluating $c_{p}, \mu$, and $\rho$, results in general model inaccuracies. Nonetheless, where possible, our model implements patient-specific vascular measurements to best approximate the patients' hepatic architecture. Due to liver-specific morphological differences and potential microvascular remodeling in Fontan livers, the model requires tuning of the parameters to patient-specific flow and pressure measurements. 


\section{Hepatic Artery Impedance}

Hepatic artery (HA) hydraulic input impedances $\left(Z_{\mathrm{HA}}\right)$ will allow for calibration of the HA tree parameters. The HA inlet pressure $\left(P_{\mathrm{HA}}\right)$ and flow signals are required to calculate $Z_{\mathrm{HA}}$ as a function of frequency $f[\mathrm{~Hz}]$. As the $P_{\mathrm{HA}}$ waveform could not be measured directly, it was approximated by scaling the magnitudes of the ascending aorta pressure waveform using systolic and diastolic brachial artery pressures. This was acceptable because the hepatic and brachial arteries both branch off from the descending aorta with a small difference in elevation at the point of measurement.

Before calculating $Z_{\mathrm{HA}}$, the phase lag between pressure and flow waveforms (which were collected at different time points, on average 1 year apart), was estimated. The flow waveform lags behind the pressure waveform due to the inertial forces preventing the blood from responding instantaneously to a change in pressure. To that end, the Womersley number $(\alpha)$ was calculated for the HA inlet as

$$
\alpha=r \sqrt{\frac{2 \pi f \rho}{\mu}}
$$

where $r$ is the inlet HA radius, and $f$ is the frequency based on patient's heart rate. Using a previously defined graph of phase lag as a function of $\alpha[30]$, the amount of lag between the peaks of flow and pressure waveforms for each patient was estimated and used when calculating $Z_{\mathrm{HA}}$. 
The HA input impedances $\left(\mathrm{Z}_{\mathrm{HA}}\right)$ were calculated by dividing the discrete Fourier transformation of $\mathrm{P}_{\mathrm{HA}}$ by that of $\mathrm{Q}_{\mathrm{HA}}$. The frequency range of the reported impedances was restricted to $0-10 \mathrm{~Hz}$, because higher frequencies are physiologically less important and may be attributed to the inherent noise of the measurements [31]. To estimate the reliability of the impedance spectra, magnitude-squared coherences $C(f)$ between pressure and flow signals were calculated [31]. These coherences quantify how well pressure and flow signals are related to each other at each frequency. $C(f)$ values are reported between 0 for nonrelated signals and 1 for perfectly related signals.

\section{Reduced Windkessel Model for HA Impedance}

Patient-specific coherence plots revealed limited frequency content showing decreasing $C(f)$ with increasing frequencies (ranging from $\sim 0.9$ at $0 \mathrm{~Hz}$ to $\sim 0.1$ at $10 \mathrm{~Hz}$ ). Frequencies with low coherence have the potential to skew the calibration results by undermining the highly coherent frequencies. Consequently, similar to the method of Debbaut et al. [31], we decided to correct for the low coherent frequencies by introducing an intermediate step in the calibration process. This entailed fitting a reduced Windkessel (WK) model configuration to the HA tree hydraulic impedance. The reduced configuration contains a $\pi$ Windkessel $(\mathrm{WK} \pi)$ model including a distal resistance $\left(R_{\mathrm{d}}\right)$, an inductor $(L)$, a capacitor $(C)$, and a component representing the viscoelasticity of the vessel walls $\left(R_{\mathrm{p}}\right)$, as shown in Figure $3 \mathrm{~b}$. The fitting procedure involved minimizing the differences between the moduli of patient-specific $Z_{\mathrm{HA}}$ and the total impedance of the reduced $\mathrm{WK} \pi$ model, $Z_{\mathrm{WK}, \mathrm{HA}}$, in the range of 0 to $10 \mathrm{~Hz}$ using the following equation

$$
\operatorname{Cost}_{W K}=\sum w\left(\left|Z_{W K, H A}\right|-\left|Z_{H A}\right|\right)^{2},
$$


where $w$ is a weighting factor. To account for coherence inconsistencies, only frequencies with $C(f)>0.5$ were included. The weight $w$ was set to the normalized coherence value for each frequency $(C(f)$ divided by the maximum coherence value in the range of $0-10 \mathrm{~Hz})$. An unconstrained nonlinear optimization method was used to fit the $Z_{\mathrm{WK}, \mathrm{HA}}$ parameters.

\section{Hepatic Artery Tree Parameter Calibration}

The HA tree is an essential part of the overall model because it contains the pressure source that is used to solve for all mean pressures. Tuning the HA tree required two separate approaches. First, we tuned the $R_{\mathrm{s}, \mathrm{HA}}$ values, which are responsible for the mean pressure drop across the HA tree, by defining a multiplying factor, $F_{R s, H A}$. This factor is multiplied by $R_{\mathrm{s}, \mathrm{HA}}$ elements of the inner generations (ones with geometries approximated by the data from Debbaut et al. [18]), to match the model predicted pressure at the end of HA tree with the pressure measured at sinusoids. Next, we defined 3 more multiplying factors, $F_{L, H A}$, $F_{C, H A}$, and $F_{R p, H A}$, that are multiplied by the $L, C$ and $R_{\mathrm{p}}$ elements of the HA tree, respectively, and are responsible for tuning the waveform features of model results. To achieve this, we calculated the overall impedance of the model with respect to the HA tree (Z $\left.Z_{\text {HA,model }}\right)$, meaning that the PV flow source was treated as an open circuit and the total impedance was calculated. The optimization process intended to minimize the differences between the moduli of previously fitted $\left|Z_{\mathrm{WK}, \mathrm{HA}}\right|$ and the $\left|Z_{\mathrm{HA} \text {,model }}\right|$ in the range $0-10 \mathrm{~Hz}$. The moduli tuning enables the estimation of the pressure and flow waveform amplitudes, which is the main objective of our study. The optimization formulation used to achieve the optimal multiplying factors, through unconstrained nonlinear optimization is defined as

$$
\operatorname{Cost}_{\text {model }}=\sum\left(\left|Z_{H A, \text { model }}\right|-\left|Z_{W K, H A}\right|\right)^{2}
$$




\section{Portal Vein and Hepatic Vein Tree Parameter Calibration}

We first tune the $R_{\mathrm{s}, \mathrm{HV}}$ values that are responsible for the mean pressure drop from the sinusoids to the HV outlet. Knowing the pressures at both of these ends allowed us to find the best $F_{R s, H V}$ values to achieve the desired pressure drop. The HV tree is known to experience minimal pressure drop in Fontan patients, mainly due to its proximity to the heightened venous pressures that induce dilation in the HV vessels and sinusoids [8]. Having approximated the radii of $\mathrm{HV}$ inner generations based on a healthy liver suggests underestimation of the vessel dilations that requires down tuning (decreasing) of the $R_{\mathrm{s}, \mathrm{HV}}$ parameters for calibration. With the PV inlet pressure being an unknown, we were unable to define $F_{R s, P V}$ in a similar manner. Consequently, we propose that $F_{R s, H V}$ could serve as an estimate for $F_{R s, P V}$. This assumption is reasonable because both vessels are veins with comparable thicknesses, which are expected to show similar resistance changes when exposed to the large venous pressure.

Lack of pressure waveforms at the PV inlet constrained us from evaluating the PV hydraulic impedances and tuning the PV tree similar to the HA tree. Therefore, we grouped the HV and PV trees as the 'venous tree' that shares the same multiplier values for calibration. Having the pressure waveform for the HV outlet allowed us to tune the HV and PV trees simultaneously through minimizing the discrepancy in the time domain waveforms of model prediction and actual patient measurements at the HV outlet. Similar to the HA tree, 3 multiplying factors, $F_{L, V}, F_{C, V}$, and $F_{R p, V}$ were defined to multiply by their corresponding circuit elements in both the PV and HV trees. The optimization procedure 
was carried out by finding optimal multiplying factors for minimizing the following cost function

$$
\operatorname{Cost}_{P_{H V}}=\sum\left(P_{H V, \text { model }}-P_{H V, \text { patient }}\right)^{2}
$$

\section{RESULTS}

\section{Patient Measurements}

Mean flow rates and pressures used for solving the model are summarized in Table 2 along with reference values from healthy non-Fontan controls. A general trend that we observed was the lower $Q_{\mathrm{PV}}$ values in group I (591 $\left.\pm 260 \mathrm{vs} .1071 \pm 253 \mathrm{ml} / \mathrm{min}\right)$, while the $Q_{\mathrm{HA}}$ values did not show specific trends, both aligned with our previous findings [7]. HV pressures were higher in group I $(20.8 \pm 3.7$ vs. $13.7 \pm 1.5 \mathrm{mmHg})$, representing higher venous pressures and possibly worse Fontan hemodynamics in general. The HVPG values were consistently low $(1 \mathrm{mmHg})$ in all patients except for patient 1 , for which $\mathrm{HVPG}=5 \mathrm{mmHg}$.

\section{Serial Resistance Calibration}

Based on Equation 5, the serial resistance $R_{s}$ has a quartic dependency on vessel radii, which is prone to large inaccuracies due to approximations made for the inner generation geometries. Therefore, calibrating $R_{\mathrm{S}}$ was an essential step to achieve more accurate estimations of $P$ PV and consequently PPG. As shown in Table $3, R_{\mathrm{s}}$ values in the HV tree required down tuning (apparent by $F_{R s, H V}$ values $\left.<1\right)$ or slight over tuning $\left(F_{R s, H V}\right.$ values slightly greater to 1) in most patients to accommodate for the negligible pressure drop of 
HVPG $=1 \mathrm{mmHg}$. Patient 1 however experienced $5 \mathrm{mmHg}$ of pressure drop and thus required almost 8 times the $R_{\mathrm{s}}$ values to account for this.

\section{Estimation of Portal Venous Pressure and Portal Pressure Gradient}

Calculated values of $P_{\mathrm{Pv}}$ and PPG are given in Table 3, along with measured values of HVPG. As hypothesized, PPG was found to be 2.9-4.0 times larger than HVPG in all patients. This range of PPG/HPVG values is comparable to the ratio of $\mathrm{PPG} / \mathrm{HVPG}=3.0$ reported by Velpula et al. [10] for a single Fontan patient with liver failure, which to the best of our knowledge is the only reported clinical measurement of both of these pressures in a Fontan patient. The major portion of PPG values are attributed to the pressure difference between the PV and sinusoids that is otherwise found to be minimal in healthy or non-Fontan patients [6], [7]. As expected, group I patients experienced larger $P_{\mathrm{PV}}$ values partly due to their already large venous pressures that are transmitted to the PV. To exclude the impact of venous pressures, PPG values were used for comparison, which demonstrated an elevated value in patient 1 . In the rest of the patients, however, the PPG values were less than $5 \mathrm{mmHg}$ and were not very different between the groups ( $3.8 \pm 0.8$ versus $4.1 \pm 0.3$ $\mathrm{mmHg}$ ). Figure 4 shows the mean pressures along all liver vascular trees calculated by the model for patient 5. In this figure, the HA pressures show a sudden decrease after the $7^{\text {th }}$ generation, while the HV and PV trees have a smoother decreasing pattern.

\section{Reduced Hepatic Artery Windkessel Model}

The impedance spectra for the HA inlet exhibited an exponentially decreasing shape (Figure 5) for all patients. The modulus value at $0 \mathrm{~Hz}$ corresponds to the total $\mathrm{HA}$ vascular 
tree resistance, which was largely elevated in two group I patients. The coherences for the depicted frequencies in Figure 5 are color coded for patients 5 and 1, which also represent the general pattern in all patients. We observe the largest $C(f)$ at $0 \mathrm{~Hz}$. The coherences tend to decrease slowly until 6-7 Hz, decrease rapidly for 7-10 Hz, increase after $10 \mathrm{~Hz}$, and repeat a similar pattern for the rest of the frequencies. The HA phase angle $\left(\varphi_{\mathrm{HA}}\right)$ has slightly negative values at the lower frequencies $(0-1 \mathrm{~Hz})$, rises sharply to positive angles from $\sim 2-5 \mathrm{~Hz}$ and then decreases rapidly to negative angles at $\sim 6-8 \mathrm{~Hz}$.

The reduced $\mathrm{WK} \pi$ model was fitted to the HA input impedances resulting in the parameter values in Table 4. Figure 5 also presents the shape of the fitted ZwK,HA for the sample patients. Overall, the fitting process seemed to provide acceptable root mean squared error (RMSEWK) values (square root of the CostwK value) despite not accounting for the lower coherent frequencies in the 7 to $10 \mathrm{~Hz}$ range. On the contrary, the phase angle plot (Figure 5) shows significant discrepancies between the reduced $\mathrm{WK} \pi$ fitted model and the $\varphi \mathrm{HA}$ values. Such observation is understandable as the WK $\pi$ models were solely fitted to the impedance moduli. Additionally, the large time gap between the flow and pressure measurements could be a source of unreliable phase patterns for the HA inlet.

\section{Total Model Calibration}

Table 5 provides values of optimal multipliers for calibrating both the HA and venous trees (PV \& HV) along with their corresponding RMSE values. We first started by fitting the $\left|Z_{\mathrm{HA}, \text { model }}\right|$ to the already fitted $\left|Z_{\mathrm{HA}, \mathrm{WK}}\right|$ by finding the optimal multiplying factors. We initially observed that several combinations of $F_{L, H A}, F_{C, H A}$, and $F_{R p, H A}$ can produce the 
same result, making the solution non-unique. Therefore, considering the dependency of $C$ and $R_{\mathrm{P}}$ on $L$ (Equations $7 \& 8$ ), we set all $F_{L, H A}$ values to 1 to constrain our solution space to a single optimal solution (Table 5) that also allows for a more intuitive comparison of $F_{C, H A}$ and $F_{R p, H A}$ among patients. The dashed lines in Figure 5 represents the final fitted $\left|Z_{\mathrm{HA}, \text { model }}\right|$ values for patients 5 and 1 , which show a slight underestimation of the $0 \mathrm{~Hz}$ impedance modulus. This modulus is controlled by $R_{\mathrm{S}}$ values that have already been calibrated and cannot be changed. Phase angles of the final calibrated model (Figure 5) seem to follow the reduced $\mathrm{WK} \pi$ model closely until $2 \mathrm{~Hz}$; this is followed by slight divergence for the rest of the frequencies. Optimal venous tree multipliers, which were based on fitting the $P_{\mathrm{HV}}$ waveform estimation with actual patient measurement, are also presented in Table 5. Similar to the HA tree, $F_{L, V}$ values were set to 1 for the calibration process.

\section{Comparison of Pressure Waveforms}

Model outputs and patient-specific measurements of $P_{H V}$ waveforms were compared, as shown in Figure 6, to determine how close the model outputs can represent the target $P_{H V}$ waveforms via parameter optimization. Qualitatively, the model can capture the main features of $P_{H V}$ for patients 1, 5, and 6, which are the patients with the TCPC Fontan connections. However, as apparent by the discrepancies in the width of the peaks of the pressure waveforms, there is less qualitative agreement for patients $2,3,4$, and 7 , which are the patients with the AP Fontan connections. Quantitatively, RMSEphv is a measure of the difference between the calculated and measured $P_{H V}$ waveforms, which was found to be smaller in the TCPC patients compared to the AP patients (RMSEPHv $=0.340$ vs. 1.513$)$. 


\section{DISCUSSION}

Fontan associated liver disease is increasingly being recognized in the adult Fontan population. Clinicians have previously relied on HVPG as a parameter for assessing the degree of liver fibrosis; however, there have been questions about its suitability as a surrogate for PPG in Fontan patients. We applied a lumped parameter model of liver hemodynamics, originally proposed by Debbaut et al. [18], to enable patient-specific estimations of $P_{P V}$ and PPG in Fontan patients, and found that HVPG underestimates PPG by a factor of 2.9 to 4.0 times across our patients, suggesting that these two pressure gradients are indeed substantially different. This ratio of PPG to HVPG was comparable to the ratio measured in a single Fontan patient. We compared transient pressure waveforms at the HV outlet and found good agreement between our model and clinically measured data for TCPC Fontan patients, as demonstrated by an average RMSEphv $=0.340$. A larger average RMSEPHv $=1.513$ found in AP Fontan patients, highlighting the limitation of the model to incorporate backflow from the right atrium, as discussed in more detail below. This model has a low computational cost of tuning and calibration (less than 30 seconds per patient), which is an ideal feature that makes it suitable for large patient cohorts.

To the best of our knowledge, the only other study that presents an LPM of the liver for Fontan patients was Kung et al. [11], which models the overall Fontan circulation including a 3-parameter model for the liver, representing its overall compliance and resistance. For non-Fontan studies, Wang et al. [21] presented a detailed LPM of the liver that uses global resistance and capacitor values to represent different liver lobes. Despite its detailed 
structure, there is limited benefit to use this model for patient-specific cases because it does not require the evaluation of circuit parameters using patient data. Our model, which builds upon the work of Debbaut et al. [18], contains detailed representations of the liver vasculature that can easily be evaluated using vessel geometries and flow waveforms, obtained non-invasively from medical images, and pressure waveforms obtained using minimally-invasive catheters. The modularity of our model also allows the development of a very detailed representation of the liver vasculature depending on the visibility of inner generations. These features make our model suitable for detailed simulation of patientspecific cases providing more accurate estimation of liver pressures. The model of Debbaut et al. [31] was calibrated based on measurements of an excised human liver and obtained simultaneous pressure and flow measurements by attaching the liver to a hypothermic machine perfusion device. Our HA hydraulic impedances had a comparable exponentially reducing pattern for the impedance moduli as a function of frequency to those found by Debbaut et al. Unlike the generally low $C(f)$ values in our patients, their results showed very large $C(f)$ values across the $0-10 \mathrm{~Hz}$ range with most of the $0-1 \mathrm{~Hz}$ and $6-10 \mathrm{~Hz}$ having $C(f)$ values greater than 0.9 . Their increased coherence values can be attributed to simultaneous collection of flow and pressure waveforms, which contrasted the clinical data in our study, for which the flow and pressure waveforms were obtained at different times. In our study, all calibrations were based on impedance moduli and there was no emphasis on simulating the phase spectra, which showed large discrepancies among the patient measurements and the calibrated model. Consequently, we did not perform a full analysis on the phase spectra. For more detail on the impact of parameter calibration on phase angles refer to Debbaut et al. [31]. 
The full calibration of model parameters was an essential step to properly simulate the patient measurements. To achieve the correct amount of pulsatility in the pressure and flow waveforms, we reduced the value of $C_{i j}$ in the majority of patients as apparent by the small values of the $F_{C}$ multiplier in Table 5 . Knowing the pulsatility of the blood is partly impacted by the liver tissue surrounding the vessels, the down tuning is indicative of the reduced compliance for the coupled liver vasculature and surrounding tissue that are caused by continuous exposure to high pressure-induced congestion. Particularly, group I patients demonstrate overall lower $F_{C, H A}$ values compared to group II patients $(0.54 \pm 0.41$ versus $0.84 \pm 0.27$ ), which emphasizes the potential role of fibrosis in reducing the compliance for the HA vascular bed and increasing the surrounding parenchymal stiffness. Venous tree multipliers did not show obvious patterns among the groups, although the low $F_{C, V}$ value of 0.121 experienced by patient 1 potentially depicts the considerably reduced venous tree compliance in cirrhotic and cancerous livers. In clinical settings, the measurement of liver parenchymal stiffness (opposite of compliance) using elastography techniques is a simple way to gain idea of the degree of liver fibrosis [32]. Our patients however lacked this measurement which would otherwise allow us to validate our calibrated $C_{i j}$ parameters. The consistently large $F_{R p}$ values across all patients (in the order of $10^{3}-10^{5}$ ) suggests significant underestimation of viscoelastic effect using the current evaluation of $R_{\mathrm{P}}$ parameter (Equation 8). It is believed that neglecting the effects of surrounding liver tissue by our method is the main reason for such underestimation, as discussed in Ref. [31]. 
A noteworthy observation is the smooth appearance of waveforms predicted by our model. Such behavior can be explained by dampening of higher frequency components that is inherent to the model solution. This model behavior may be a limiting factor for obtaining more comparable waveforms to patient measurements (Figure 6) especially because many of the measured $\mathrm{HV}$ outlet pressures contain higher frequency features (small peaks and troughs along the waveform) that our model cannot encompass. That being said, the inherent noise associated with pressure transducers can be a confounding variable, introducing unwanted higher frequency components to the measurements. The significantly narrower peaks observed in AP patient measurements, which are influenced by the atrial contraction, lead to large RMSEphv values in these patients. A combination of the elevated pressure due to buildup of static blood volume in the right atrium coupled with the atrial contraction induces a highly pulsatile pressure to the IVC blood that in turn results in large amount of backflow into the HV tree [3]. The modified hemodynamics at the liver outlet is likely due to the impact of the right atrium, which is outside the scope for our model. Therefore, assuming the continuity of flow and a pressure driven fluid motion from liver inlet to the outlet contradicts with the observation of backflow into the liver vasculature and cannot provide an accurate representation of hepatic hemodynamics in AP patients.

There are several limitations to this study. Despite the detailed nature of our model, it assumes simplified branching patterns that are mostly observed in healthy livers. In Fontan patients, incidence of vascular shunts are not uncommon, especially in cirrhotic livers experiencing large PPG [3]. These vascular connections introduce new pathways for blood 
flow in and around the liver that can impact the pressure drops and liver flow volumes. We did not observe any shunts among our patients; however, having access to high resolution CT images would be useful in detecting such connections, which could be implemented in the model by the addition of bridging components connecting the corresponding generations. Lacking liver specific imaging in our patients meant that we could only use the liver images in the PC-CMR scans for our geometric measurements. Due to the low image resolution coupled with the available number of slices, we could only visualize vessels up to the $3^{\text {rd }}$ generation for all of our patients. Therefore, we complemented the data generated by this visualization with the measurements from Debbaut et al. [18] of a single healthy non-Fontan liver. As an implication of this limitation, the inner PV and HV serial resistances would stay constant across all our patients, hence limiting the personalization of PPG estimations. To mitigate this partially, patient specific flow rates ( $Q_{\mathrm{PV}}$ and $\left.Q_{\mathrm{HV}}\right)$, HVPG, and $F_{\mathrm{Rs}, \mathrm{HV}}$ values were implemented for the calculation of PPG estimates that resulted in wide ranges of values across our patients. Having high resolution CT imaging of liver would allow us to measure further inner generations (up to 5 or more) that can later be used to extrapolate the geometries for much smaller generations up until the level of sinusoids.

The definitive way to verify the model predictions would be by experimental means, similar to the approach of Debbaut et al. [31]. Attaching an excised Fontan liver to a perfusion machine would enable pressure measurement at various locations, particularly the inlets and outlet of the liver. Running experiments under several flow and pressure conditions and comparing the results with model outputs would serve as an ideal verifier 
for the model's predictive capability. In the clinical setting, some Fontan patients with liver failure/cancer who require interventions also undergo direct measurements of $P$ Pv and PPG as in the case study by Velpula et al. [10]. Testing the model on Fontan patients with available $P_{\mathrm{PV}}$ and PPG data could also verify our model's ability to predict PPG in a wide range of Fontan livers.

Ideally, the pressure and flow measurement would be performed simultaneously, which is unpractical in clinical settings due to the different requirements for PC-CMR and cardiac catheterization procedures, or at least on the same-day, to enhance the coherence values between the two. Unfortunately, the mean time difference for our patients was about 1 year, suggesting two different liver health states, which would result in lower coherence values. Liver biopsy procedure is the gold standard for detection of cirrhosis and evaluating the degree of fibrosis in clinical settings; however, their invasive nature makes them an uncommon addition to routine follow-ups [32]. Ideally, having access to these values would allow for grading the severity of liver disease in our patients and developing a new scale for interpreting the clinical significance of PPG values.

\section{Conclusions}

A detailed liver lumped parameter model proposed by Debbaut et al. has been applied to seven Fontan patients, yielding good agreement to clinical data, particularly for patients with TCPC Fontan connections. This model illustrates the discrepancy between portal pressure gradient (PPG) and hepatic venous pressure gradient in this patient population, highlighting the unsuitability of using the latter as a surrogate for the former in Fontan 
patients. With limited patient-specific geometric data, claiming a high degree of accuracy for PPG estimates and therefore recommendation for use in clinical settings is not possible. However, with future studies taking advantage of better imaging and possible validation using actual PPG measurements, it is believed that such models could be used in clinical settings to monitor patient's PPG and liver compliance over consecutive follow-ups, determine the progression of liver disease, and allow for improved patient management.

\section{Funding}

Natural Sciences and Engineering Research Council of Canada (NSERC) Canadian

Graduate Scholarship-Master's (CGS-M) (EAB), postdoctoral fellowship from the Research Foundation - Flanders (FWO), Belgium (CD) (1202418N), NSERC Grant (CHA).

\section{Conflict of Interest}

none

\section{Nomenclature}

$C_{i j} \quad$ Parallel capacitance

$C(f) \quad$ Coherence values at a certain frequency

$c_{p} \quad$ Pulse wave velocity

E $\quad$ wall $\quad$ Young's modulus

F Multiplying factor

$f \quad$ Frequency 


\begin{tabular}{ll}
$h_{\text {wall }}$ & Wall thickness \\
$j$ & Generation number \\
$L_{i j}$ & Serial inductance \\
$l$ & Vessel length \\
$N$ & Number of vessels per generation \\
$P$ & Pressure \\
$Q$ & Flow rate \\
$r$ & Vessel radius \\
$R_{d}$ & Distal resistance \\
$R_{s i j}$ & Serial resistance \\
$R_{p i j}$ & Parallel resistance \\
$Z$ & Impedance \\
$\alpha$ & Womersley number \\
$\rho$ & Blood density \\
$\mu$ & Dynamic blood viscosity \\
$\mu_{\text {wall }}$ & Wall viscoelasticity \\
\hline &
\end{tabular}

\section{REFERENCES}

[1] Munsterman, I.D., Duijnhouwer, A.L., Kendall, T.J., Bronkhorst, C.M., Ronot, M., van Wettere, M., van Dijk, A.P.J, Drenth, J.P.H., and Tjwa, E.T.T.L., 2018, “The Clinical Spectrum of Fontan-Associated Liver Disease: Results from a Prospective Multimodality Screening Cohort." European Heart Journal, 40 (13), pp. 1057-68. doi:10.1093/eurheartj/ehy620.

[2] Schwartz, M.C., Sullivan, L., Cohen, M.S., Russo, P., John, A.S., Guo, R., Guttenberg, M. and Rand, E.B., 2012, "Hepatic Pathology May Develop before the Fontan Operation in Children with Functional Single Ventricle: An Autopsy Study." 
Journal of Thoracic and Cardiovascular Surgery, 143(4), pp. 904-9. doi:10.1016/j.jtcvs.2011.08.038.

[3] Wells, M.L., Fenstad, E.R., Poterucha, J.T., Hough, D.M., Young, P.M., Araoz, P.A., Ehman, R.L., and Venkatesh. S.K., 2016, "Imaging Findings of Congestive Hepatopathy." RadioGraphics, 36(4), pp. 1024-37. doi:10.1148/rg.2016150207.

[4] Abbasi Bavil, E., Yang, H.K., Doyle, M.G., Kim, T.K., Karur, G.R., Bhagra, C., Bhagra, S., Oechslin, E.N., Ross, H.J., Roche, S.L., Gonzalez, R.A., Amon, C.H., Mertens, L., and Wald, R.M., 2020, "Cardiovascular and Abdominal Flow Alterations in Adults with Morphologic Evdence of Liver Disease Post Fontan Palliation," Int. J. Cardiol., 317, pp.63-9. doi:10.1016/j.ijcard.2020.05.064.

[5] Shetty, S., Lalor, P.F., and Adams, D.H., 2018, "Liver Sinusoidal Endothelial Cells - Gatekeepers of Hepatic Immunity." Nature Reviews Gastroenterology and Hepatology, 15(9), pp. 555-67. doi:10.1038/s41575-018-0020-y.

[6] Suk, K.T., 2014, "Hepatic Venous Pressure Gradient: Clinical Use in Chronic Liver Disease." Clinical and Molecular Hepatology, 20(1), pp. 6-14. doi:10.3350/cmh.2014.20.1.6.

[7] Reynolds, T.B., Ito, S., and Iwatsuki, S., 1970, "Measurement of Portal Pressure and Its Clinical Application." The American Journal of Medicine, 49(5), pp. 649-57. doi:10.1016/S0002-9343(70)80131-0.

[8] Kendall, T.J., Stedman, B., Hacking, N., Haw, M., Vettukattill, J.J., Salmon, A.P., and Cope, R., 2008, "Hepatic Fibrosis and Cirrhosis in the Fontan Circulation: A Detailed Morphological Study.” Journal of Clinical Pathology, 61(4), pp. 504-8. doi:10.1136/jcp.2007.052365.

[9] Téllez, L., de Santiago, E.R., and Albillos, A., 2018, "Fontan-Associated Liver Disease." Revista Espanola de Cardiologia, 17(2), pp. 192-202. doi:10.1016/j.recesp.2017.10.014.

[10] Velpula, M., Sheron, N., Guha, N., Salmon, T., Hacking, N., and Veldtman, G.R., 2011, "Direct Measurement of Porto-Systemic Gradient in a Failing Fontan Circulation." Congenital Heart Disease 6(2), pp. 175-78. doi:10.1111/j.17470803.2010.00451.x.

[11] Kung, E., Pennati, G., Migliavacca, F., Hsia, T.V., Figliola, R., Marsden, A., and Giardini, A., 2014, "A Simulation Protocol for Exercise Physiology in Fontan Patients Using a Closed Loop Lumped-Parameter Model." Journal of Biomechanical Engineering, 136(8), pp. 081007. doi:10.1115/1.4027271.

[12] Watrous, R.L., and Chin, A.J., 2014, "Model-Based Comparison of the Normal and Fontan Circulatory Systems: Part I: Development of a General Purpose, Interactive Cardiovascular Model." World Journal for Pediatric and Congenital Heart Surgery, 5(3), pp. 372-84. doi:10.1177/2150135114529450. 
[13] Koeken, Y., Arts, T., and Delhaas, T., 2012, "Simulation of the Fontan Circulation during Rest and Exercise." In Proceedings of the Annual International Conference of the IEEE Engineering in Medicine and Biology Society, EMBS, 6673-76. doi:10.1109/EMBC.2012.6347525.

[14] Chugunova, M., Doyle, M.G., Keener, J.P., and Taranets, R.M., 2018, "Use of Mathematical Modeling to Study Pressure Regimes in Normal and Fontan Blood Flow Circulations.” Mathematics-in-Industry Case Studies, doi:10.1186/s40929019-0019-9.

[15] Debbaut, C., Vierendeels, J., Casteleyn, C., Cornillie, P., Loo, D.V., Simoens, P., Van Hoorebeke, L., Monbaliu, D., and Segers, P., 2012, "Perfusion Characteristics of the Human Hepatic Microcirculation Based on Three-Dimensional Reconstructions and Computational Fluid Dynamic Analysis." Journal of Biomechanical Engineering, 134(1), pp. 011003-10. doi:10.1115/1.4005545.

[16] Ho, C.M., Lin, R.K., Tsai, S.F., Hu, R.H., Liang, P.C., Sheu, T.W.H., and Lee, P.H., 2010, "Simulation of Portal Hemodynamic Changes in a Donor after Right Hepatectomy." Journal of Biomechanical Engineering, 132(4), pp. 041002-07 doi:10.1115/1.4000957.

[17] Peeters, G., Debbaut, C., Cornillie, P., De Schryver, T., Monbaliu, D., Laleman, W., and Segers, P., 2015, "A Multilevel Modeling Framework to Study Hepatic Perfusion Characteristics in Case of Liver Cirrhosis." Journal of Biomechanical Engineering, 137(5), pp. 051007-09. doi:10.1115/1.4029280.

[18] Debbaut, C., Monbaliu, D., Casteleyn, C., Cornillie, P., Loo, D.V., Masschaele, B., Pirenne, J., Simoens, P., Hoorebeke, L.V., and Segers, P., 2011, "From Vascular Corrosion Cast to Electrical Analog Model for the Study of Human Liver Hemodynamics and Perfusion." IEEE Transactions on Biomedical Engineering, 58(1), pp. 25-35. doi:10.1109/TBME.2010.2065229.

[19] Plaats, A.V., Hart, N.A., Verkerke, G.J., Leuvenink, H.G.D., Verdonck, P., Ploeg, R.J., and Rakhorst, G., 2004, "Numerical Simulation of the Hepatic Circulation." International Journal of Artificial Organs, 27(3), pp. 222-30.

[20] Audebert, C., Peeters, G., Segers, P., Laleman, W., Monbaliu, D., Korf, H., Trebicka, J., Vignon-Clementel, I.E., and Debbaut, C., 2018, 'Closed-Loop Lumped Parameter Modeling of Hemodynamics during Cirrhogenesis in Rats." IEEE Transactions on Biomedical Engineering, 65(10), pp. 2311-22. doi:10.1109/TBME.2018.2793948.

[21] Wang, T., Liang, F., Zhou, Z., and Shi, L., 2017, “A Computational Model of the Hepatic Circulation Applied to Analyze the Sensitivity of Hepatic Venous Pressure Gradient (HVPG) in Liver Cirrhosis." Journal of Biomechanics, 65, pp. 23-31. doi:10.1016/j.jbiomech.2017.09.023. 
[22] Fredenburg, T.B., Johnson, T.R., and Cohen, M.D., 2011, "The Fontan Procedure: Anatomy, Complications, and Manifestations of Failure." RadioGraphics, 31(2), pp. 453-63. doi:10.1148/rg.312105027.

[23] Abdel-Misih, S.R.Z., and Bloomston, M., 2010, "Liver Anatomy." Surgical Clinics of North America 90(4), pp. 643-53. doi:10.1016/j.suc.2010.04.017.

[24] de Pater, L., and van den Berg, J., 1964, "An Electrical Analogue of the Entire Human Circulatory System.” Medical Electronics \& Biological Engineering, 2(2), pp. 2161-66. doi:10.1007/BF02484215.

[25] George, S.M., Eckert, L.M., Martin, D.R., and Giddens, D.P., 2015, "Hemodynamics in Normal and Diseased Livers: Application of Image-Based Computational Models." Cardiovascular Engineering and Technology, 6(1), pp. 8091. doi:10.1007/s13239-014-0195-5.

[26] Rani, H.P., Sheu, T.W.H., Chang, T.M., and Liang, P.C., 2006, "Numerical Investigation of Non-Newtonian Microcirculatory Blood Flow in Hepatic Lobule." Journal of Biomechanics, 39(3), pp. 551-63. doi:10.1016/j.jbiomech.2004.11.029.

[27] Wang, P.J., Li, W.C., Xi, G.M., Wang, H.Q., Zhang, Z.H., Yao, B.C., Tang, W., Deng, Z.H., and Zhang, X.H., 2009, "Biomechanical Study of Hepatic Portal Vein in Humans and Pigs and Its Value in Liver Transplantation." Transplantation Proceedings, 41(5), pp. 1906-10. doi:10.1016/j.transproceed.2008.10.097.

[28] Wachsberg, R.H., Angyal, E.A., Klein, K.M., Kuo, H.R., and Lambert, W.C., 1997, "Echogenicity of Hepatic versus Portal Vein Walls Revisited with Histologic Correlation." Journal of Ultrasound in Medicine, 16(12), pp. 807-10. doi:10.7863/jum.1997.16.12.807.

[29] Willemet, M., Chowienczyk, P., and Alastruey, J., 2015, "A Database of Virtual Healthy Subjects to Assess the Accuracy of Foot-to-Foot Pulse Wave Velocities for Estimation of Aortic Stiffness." American Journal of Physiology-Heart and Circulatory Physiology, 309(4), pp. H663-75. doi:10.1152/ajpheart.00175.2015.

[30] Truskey, G.A, Yuan, F., Katz, D.F., 2009, "Transport Phenomena in Biological Systems." 2nd ed., New Jersey: Pearson.

[31] Debbaut, C., Monbaliu, D.R.L., and Segers. P., 2014, "Validation and Calibration of an Electrical Analog Model of Human Liver Perfusion Based on Hypothermic Machine Perfusion Experiments." International Journal of Artificial Organs, 37(6), pp. 486-98. doi:10.5301/ijao.5000337.

[32] Kutty, S.S., Peng, Q., Danford, D.A., Fletcher, S.E., Perry, D., Talmon, G.A., Scott, C., Kugler, J.D., Duncan, K.F., Quiros-Tejeira, R.E., Kutty, S., 2014, “Increased Hepatic Stiffness as Consequence of High Hepatic Afterload in the Fontan Circulation: A Vascular Doppler and Elastography Study." Hepatology 59(1), pp. 251-60. doi:10.1002/hep.26631. 
[33] Mcavoy, N.C., Semple, S., Richards, J.M., Robson, A.J., Patel, D., Jardine, A.G., Leyland, K., Cooper, A.S., Newby, D.E., Hayes, P.C., 2016, "Differential visceral blood flow in the hyperdynamic circulation of patients with liver cirrhosis."

Aliment. Pharmacol. Ther. 43(9), pp. 947-54. doi:10.1111/apt.13571.

[34] Hadaegh, F., Shafiee, G., Hatami, M., Azizi, F., 2012, "Systolic and diastolic blood pressure, mean arterial pressure and pulse pressure for prediction of cardiovascular events and mortality in a Middle Eastern population." Blood Press. 21(1), pp. 12-8. doi:10.3109/08037051.2011.585808.

[35] Lebrec, D., Sogni, P., Vilgrain, V., 1997, "Evaluation of patients with portal hypertension.” Baillières Clin. Gastroenterol. 11(2), pp. 221-41. doi:10.1016/s0950-3528(97)90037-3.

[36] Bari, K., \& Garcia-Tsao, G., 2012. "Treatment of portal hypertension.” World J Gastroenterol. 18(11), pp. 1166-75. doi:10.3748/wjg.v18.i11.1166. 


\section{Figure Caption List}

Figure $1 \quad$ (a) Simplified vascular anatomy highlighting the vessels and locations for collection of pressure and flow waveforms. (b) Phase contrast flow analysis demonstrating cross-sectional views of the portal vein (PV) and hepatic artery (HA) with regions of interest drawn along the vessel perimeter to allow for calculation of flow rate $(\mathrm{mL} / \mathrm{min})$.

Figure 2 Methodological approach to obtain and calibrate a liver-specific lumped parameter model for Fontan patients.

Figure 3 (a) Lumped parameter model of a Fontan liver based on the model of Debbaut et al. [18], (b) reduced configurations of a $\pi$ Windkessel model, and (c) representation of a transmission line of length $\Delta \mathrm{z}$.

Figure 4 Mean pressures across the generations of the liver vasculature for patient 5. Hepatic artery (HA) tree is generations 1 to 18 , portal vein $(\mathrm{PV})$ tree is generations 3 to 18 , and hepatic vein $(\mathrm{HV})$ tree is generations 19 to 31 .

Figure $5 \quad$ Hepatic artery (HA) impedance moduli and phase angle values between 0 to $10 \mathrm{~Hz}$ along with the best fitted reduced $\pi$ Windkessel model (WK $\pi$ ) and electrical model impedances. Panels (a) and (b) present the spectra for patient 5, and (c) and (d) for patient 1 . 
Figure 6 Comparison of hepatic vein outlet pressures from the model and patient (Pt.) measurements, where patients 1, 5, and 6 have TCPC anatomies and patients 2, 3, 4, and 7 have AP anatomies. 


\section{Table Caption List}

Table 1

Table 2

Table 3

Table 4

Table 5
Patient demographics.

Summary of measured mean flow rates and pressures along with reference values for healthy non-Fontan controls.

$R_{\mathrm{s}}$ multiplier values and model prediction for $P$ PV and PPG.

Parameters for reduced $\mathrm{WK} \pi$ model fitted to the $Z_{\text {HA }}$ modulus.

Optimal multiplier values used for tuning the model parameters. 
a.
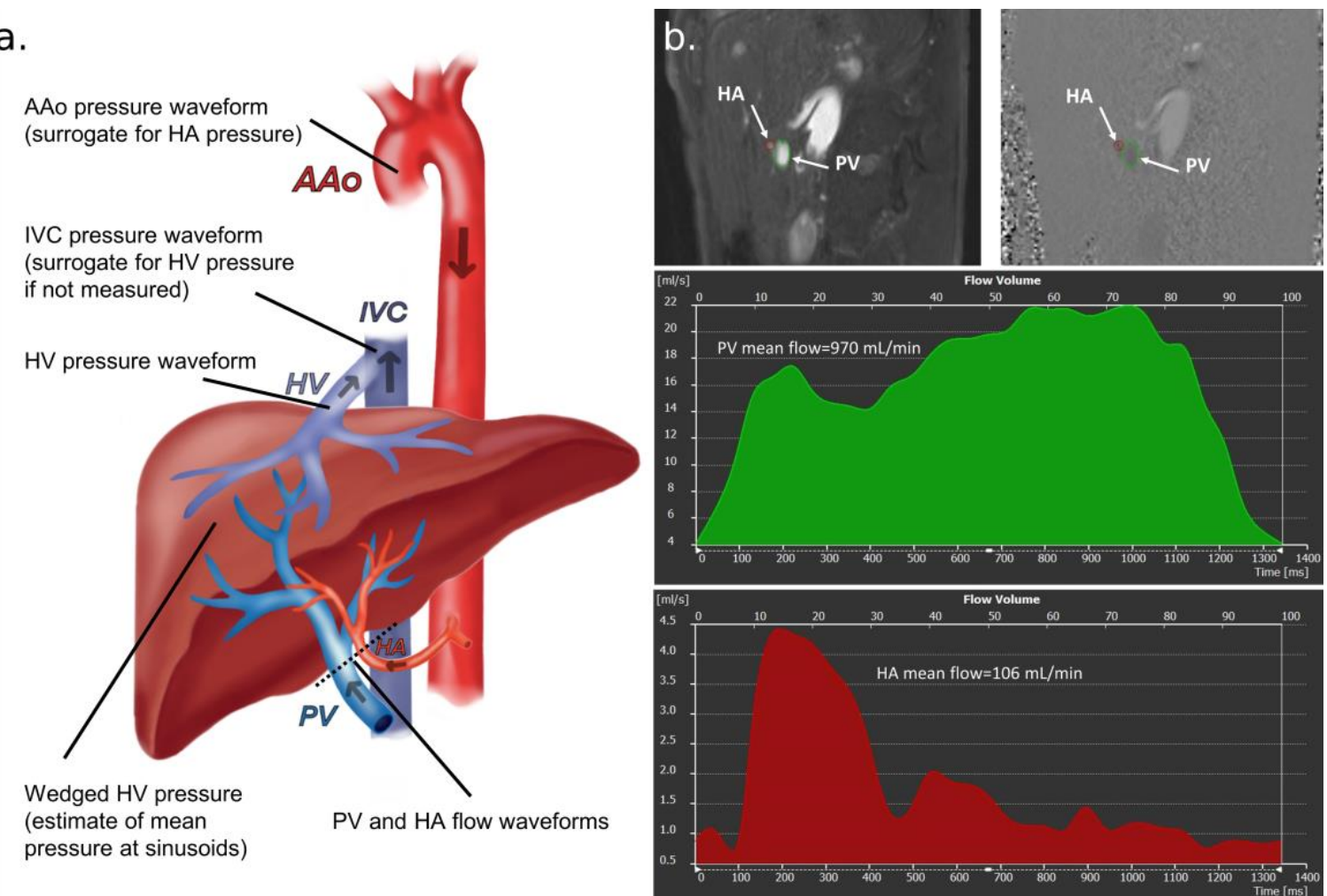

Figure 1. (a) Simplified vascular anatomy highlighting the vessels and locations for collection of pressure and flow waveforms. (b) Phase contrast flow analysis demonstrating cross-sectional views of the portal vein (PV) and hepatic artery (HA) with regions of interest drawn along the vessel perimeter to allow for calculation of flow rate. 


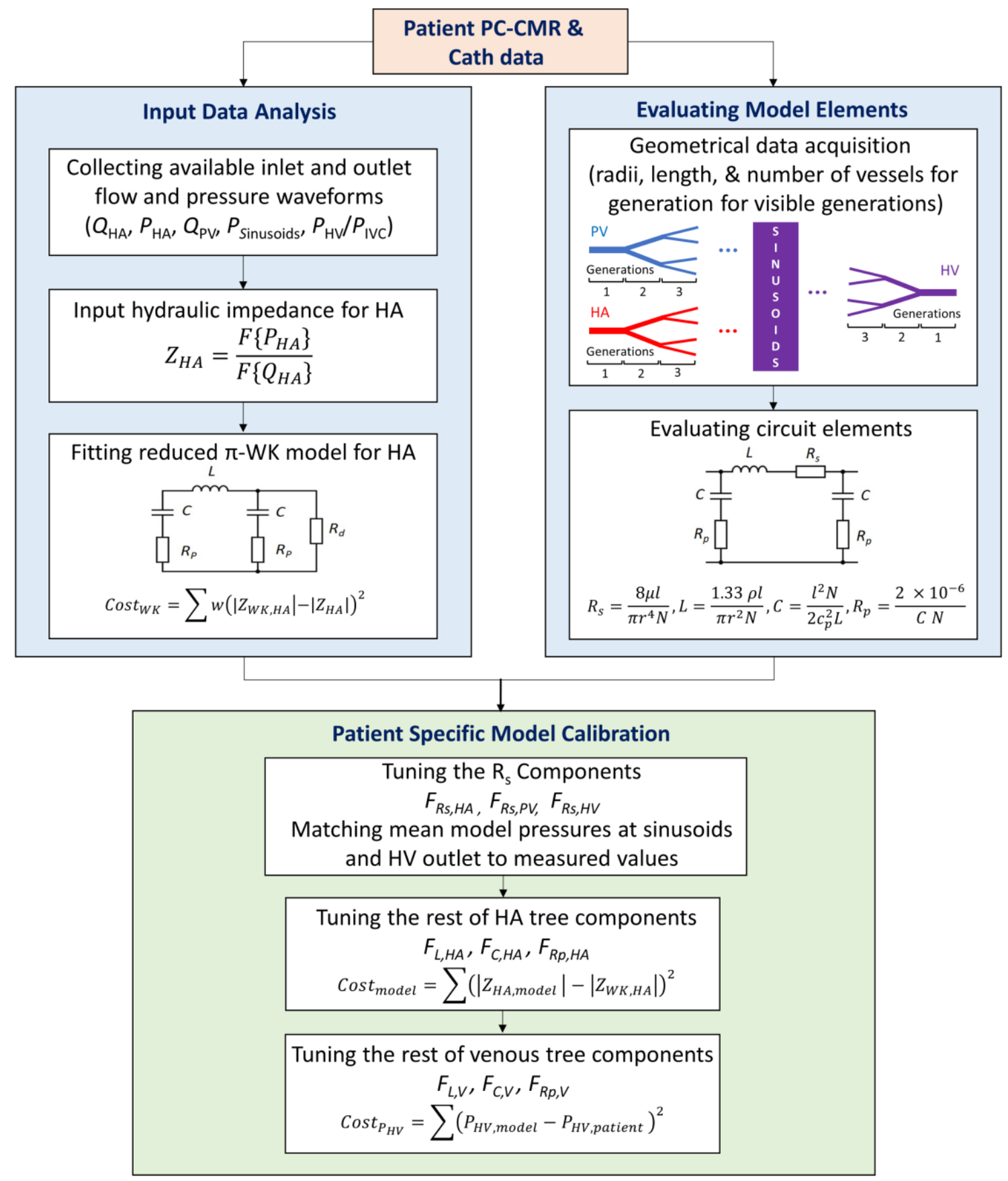

Figure 2. Methodological approach to obtain and calibrate a liver-specific lumped parameter model for Fontan patients. 

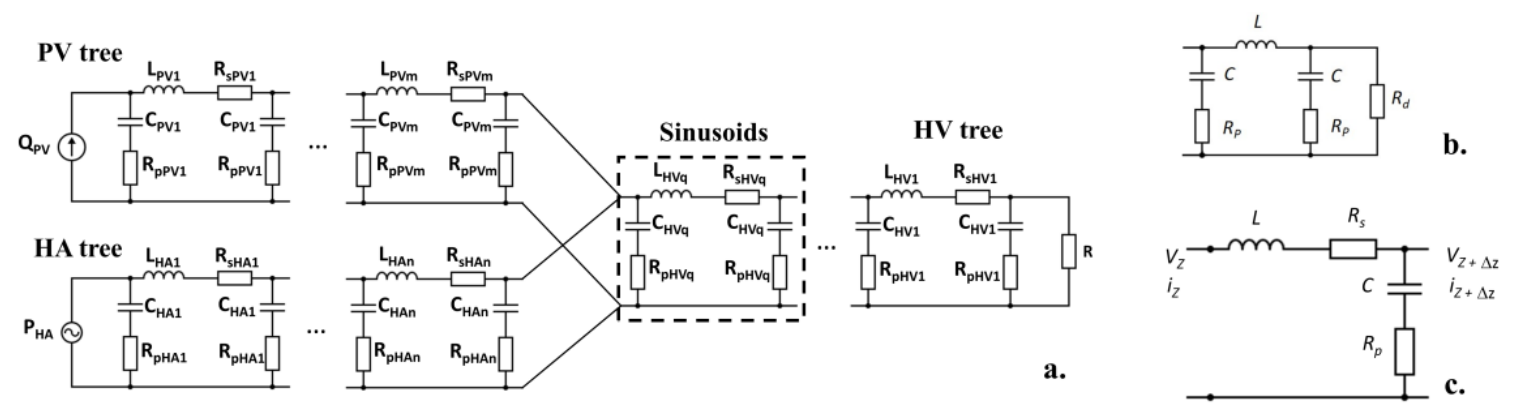

Figure 3. (a) Lumped parameter model of a Fontan liver based on the model of Debbaut et al. [18], (b) reduced configurations of a $\pi$-Windkessel model, and (c) representation of a transmission line of length $\Delta \mathrm{z}$. 


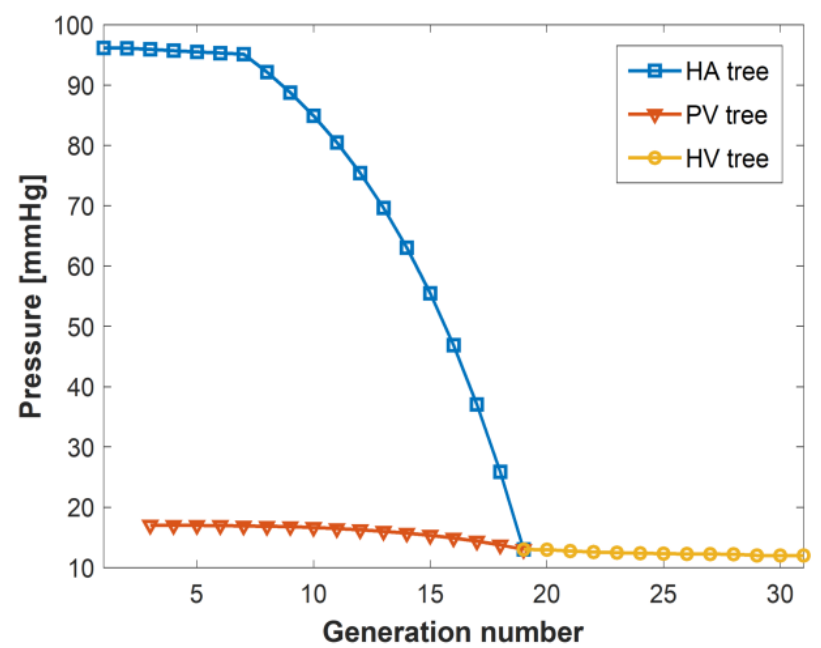

Figure 4. Mean pressures across the generations of the liver vasculature for patient 5.

Hepatic artery (HA) tree is generations 1 to 18 , portal vein (PV) tree is generations 3 to 18 , and hepatic vein $(\mathrm{HV})$ tree is generations 19 to 31 . 
a.

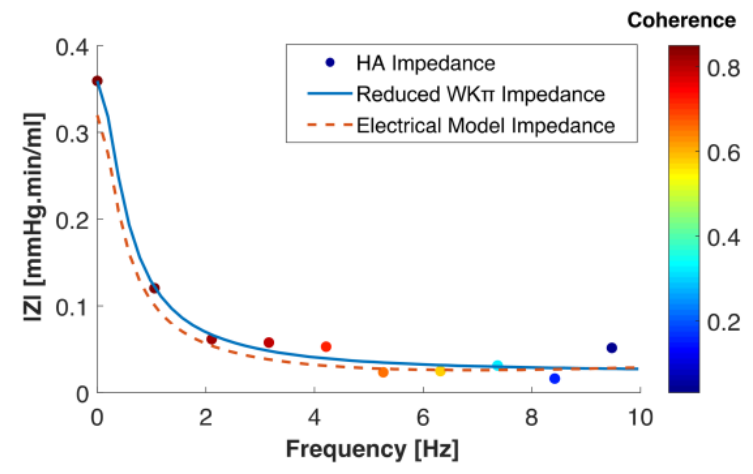

c.

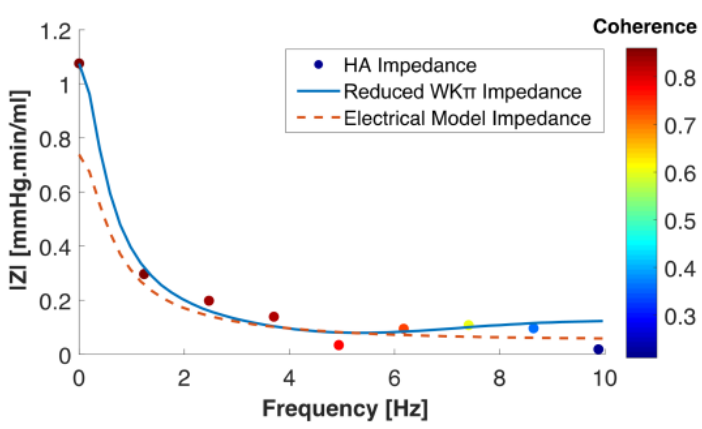

b.

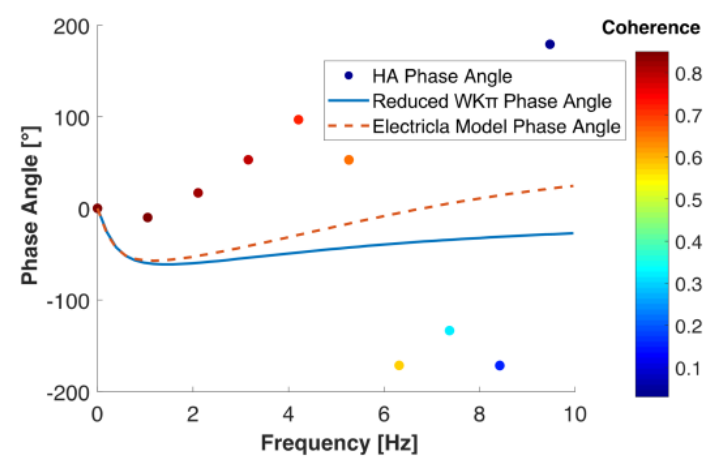

d.

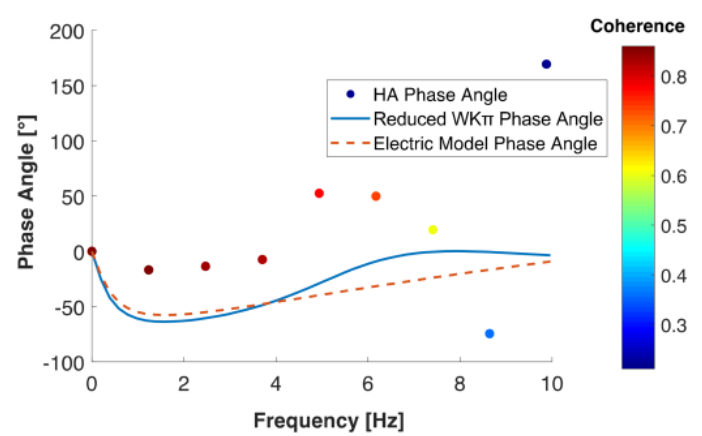

Figure 5. Hepatic artery (HA) impedance moduli and phase angle values between 0 to 10 $\mathrm{Hz}$ along with the best fitted reduced $\pi$-Windkessel model $(\mathrm{WK} \pi)$ and electrical model impedances. Panels (a) and (b) present the spectra for patient 5, and (c) and (d) for patient 1. 

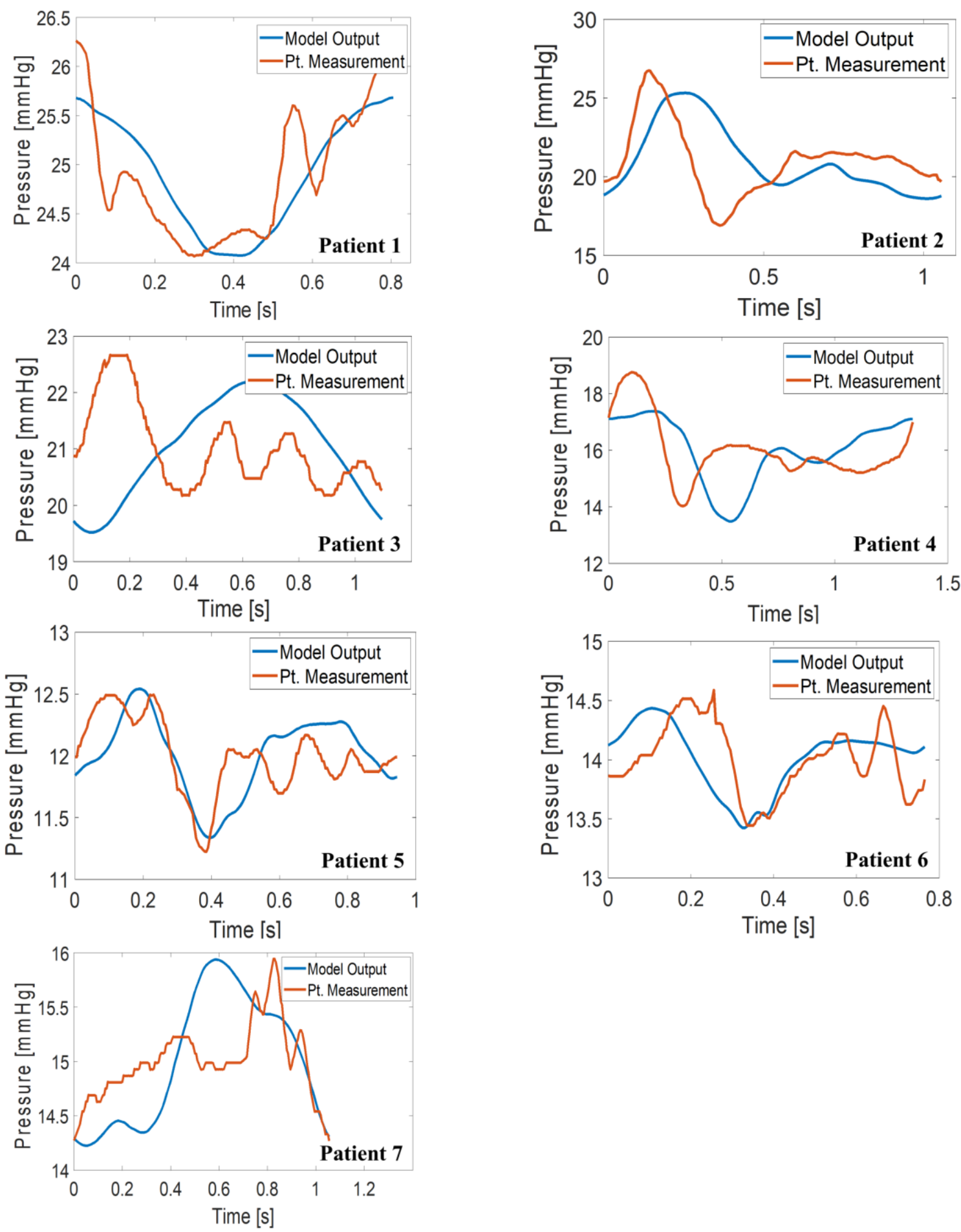

Figure 6. Comparison of hepatic vein outlet pressures from the model and patient (Pt.) measurements, where patients 1,5, and 6 have TCPC anatomies and patients 2, 3, 4, and 7 have AP anatomies. Note that both pressure and time are presented with different scales in different graphs. 
Table 1. Patient demographics.

\begin{tabular}{cccc}
\hline $\begin{array}{c}\text { Patient } \\
\text { Number }\end{array}$ & Fontan Type & $\begin{array}{c}\text { Age at } \\
\text { PC-CMR } \\
\text { (years) }\end{array}$ & Sex \\
\hline \multicolumn{4}{c}{ Group I } \\
1 & Lateral tunnel & 37 & $\mathrm{~F}$ \\
2 & Atriopulmonary & 46 & $\mathrm{~F}$ \\
3 & Atriopulmonary & 24 & $\mathrm{M}$ \\
4 & Atriopulmonary & 29 & $\mathrm{M}$ \\
5 & Group II & & \\
5 & Lateral tunnel & 25 & $\mathrm{M}$ \\
6 & Extracardiac & 39 & $\mathrm{~F}$ \\
7 & Atriopulmonary & 39 & $\mathrm{~F}$ \\
\hline
\end{tabular}


Table 2. Summary of measured mean flow rates and pressures along with reference values for healthy non-Fontan controls.

\begin{tabular}{|c|c|c|c|c|c|}
\hline $\begin{array}{c}\text { Pt. } \\
\#\end{array}$ & $\underset{[\mathrm{ml} / \mathrm{min}]}{\boldsymbol{Q}_{\mathbf{P V}}}$ & $\underset{[\mathrm{ml} / \mathrm{min}]}{\boldsymbol{Q}_{\mathbf{H A}}}$ & $\begin{array}{c}\boldsymbol{P}_{\mathbf{H A}} \\
{[\mathrm{mmHg}]} \\
(\text { Sys, Dias })\end{array}$ & $\begin{array}{c}\boldsymbol{P}_{\mathbf{H V}} \\
{[\mathrm{mmHg}]}\end{array}$ & $\begin{array}{c}\text { HVPG } \\
{[\mathrm{mmHg}}\end{array}$ \\
\hline \multicolumn{6}{|c|}{ Group I } \\
\hline 1 & 410 & 84 & $90(118 / 70)$ & 25 & 5 \\
\hline 2 & 543 & 584 & $86(112 / 67)$ & 21 & 1 \\
\hline 3 & 440 & 90 & $\begin{array}{c}114 \\
(158 / 78)\end{array}$ & 21 & 1 \\
\hline 4 & 970 & 106 & $\begin{array}{c}109 \\
(142 / 89)\end{array}$ & 16 & 1 \\
\hline \multicolumn{6}{|c|}{ Group II } \\
\hline 5 & 1063 & 268 & $96(129 / 71)$ & 12 & 1 \\
\hline 6 & 822 & 309 & $88(122 / 68)$ & 14 & 1 \\
\hline 7 & 1328 & 240 & $84(101 / 72)$ & 15 & 1 \\
\hline \multicolumn{6}{|c|}{ Healthy non-Fontan controls } \\
\hline & $776 \pm 349[33]$ & $251 \pm 148$ [33] & $91[34]$ & $2-7[35]$ & $3-5[36]$ \\
\hline
\end{tabular}


Table 3. $R_{\mathrm{s}}$ multiplier values and model prediction for $P$ PV and PPG.

\begin{tabular}{lccccc}
\hline Pt. \# & $\boldsymbol{F}_{\boldsymbol{R} \boldsymbol{s}, \boldsymbol{H A}}$ & $\boldsymbol{F}_{\boldsymbol{R} \boldsymbol{s}, \boldsymbol{H V}}$ & $\begin{array}{c}\boldsymbol{P} \mathbf{P V} \\
{[\mathrm{mmHg}]} \\
\text { mean }\end{array}$ & $\begin{array}{c}\text { PPG } \\
{[\mathrm{mmHg}]} \\
\text { mean }\end{array}$ & $\begin{array}{c}\text { HVPG } \\
{[\mathrm{mmHg}]} \\
\text { mean }\end{array}$ \\
\hline \multicolumn{5}{c}{ Group I } \\
1 & 3.036 & 8.255 & 45.2 & 20.2 & 5 \\
2 & 0.464 & 0.724 & 23.9 & 2.9 & 1 \\
3 & 4.315 & 1.541 & 25.2 & 4.2 & 1 \\
4 & 2.282 & 0.710 & 20.3 & 4.3 & 1 \\
& & & Group II & & \\
5 & 1.311 & 0.662 & 16.4 & 4.4 & 1 \\
6 & 0.996 & 0.690 & 17.8 & 3.8 & 1 \\
7 & 1.201 & 0.499 & 19.2 & 4.2 & 1 \\
\hline
\end{tabular}


Table 4. Parameters for reduced WK $\pi$ model fitted to the ZHA modulus.

\begin{tabular}{|c|c|c|c|c|c|c|}
\hline Pt. \# & $\begin{array}{c}\mathrm{Z}_{\mathrm{HA}}(\mathbf{O ~ H z}) \\
{[\mathrm{mmHg} .} \\
\mathrm{min} / \mathrm{ml}]\end{array}$ & $\begin{array}{c}\boldsymbol{R}_{\mathbf{d}} \\
{[\mathrm{mmHg} \cdot} \\
\mathrm{min} / \mathrm{ml}]\end{array}$ & $\begin{array}{c}\boldsymbol{L} \\
{\left[\mathrm{mmHg}^{2}\right.} \\
\left.\mathrm{min}^{2} / \mathrm{ml}\right]\end{array}$ & $\begin{array}{c}\boldsymbol{C} \\
{[\mathrm{ml} / \mathrm{mmHg}]}\end{array}$ & $\begin{array}{c}\boldsymbol{R}_{\mathbf{p}} \\
{[\mathrm{mmHg} .} \\
\mathrm{min} / \mathrm{ml}]\end{array}$ & RMSEWK \\
\hline \multicolumn{7}{|c|}{ Group I } \\
\hline 1 & 1.07 & 1.075 & $3.900 \times 10^{-3}$ & 0.181 & 0.103 & 0.0053 \\
\hline 2 & 0.15 & 0.147 & $1.367 \times 10^{-6}$ & 0.709 & 0.064 & 0.0007 \\
\hline 3 & 1.27 & 1.172 & $8.600 \times 10^{-3}$ & 0.046 & 0.006 & 0.1837 \\
\hline 4 & 0.64 & 0.640 & $1.300 \times 10^{-3}$ & 0.429 & 0.097 & 0.0035 \\
\hline \multicolumn{7}{|c|}{ Group II } \\
\hline 5 & 0.36 & 0.310 & $2.589 \times 10^{-6}$ & 0.557 & 0.051 & 0.0004 \\
\hline 6 & 0.28 & 0.284 & $4.630 \times 10^{-4}$ & 0.596 & 0.057 & 0.0006 \\
\hline 7 & 0.35 & 0.351 & $9.508 \times 10^{-6}$ & 1.554 & 0.032 & 0.0004 \\
\hline
\end{tabular}


Table 5. Optimal multiplier values used for tuning the model parameters.

\begin{tabular}{lcccccc}
\hline Pt. \# & $\boldsymbol{F}_{\boldsymbol{C}, \boldsymbol{H A}}$ & $\boldsymbol{F}_{\boldsymbol{R} \boldsymbol{H}, \boldsymbol{H} \boldsymbol{A}}$ & $\mathbf{R M S E m o d e l ~}$ & $\boldsymbol{F}_{\boldsymbol{C}, \boldsymbol{V}}$ & $\boldsymbol{F}_{\boldsymbol{R} p, \boldsymbol{V}}$ & RMSEPHV \\
\hline \multicolumn{5}{c}{} \\
1 & 0.323 & $9.820 \times 10^{3}$ & 0.099 & 0.121 & $1.210 \times 10^{4}$ & 0.419 \\
2 & 0.976 & $1.529 \times 10^{4}$ & 0.005 & 0.069 & $1.200 \times 10^{4}$ & 2.699 \\
3 & 0.078 & $9.105 \times 10^{3}$ & 0.117 & 0.138 & $1.100 \times 10^{4}$ & 1.513 \\
4 & 0.772 & $2.041 \times 10^{4}$ & 0.022 & 0.875 & $1.550 \times 10^{5}$ & 1.339 \\
& & & Group II & & \\
5 & 0.964 & $1.266 \times 10^{4}$ & 0.012 & 0.222 & $2.347 \times 10^{4}$ & 0.286 \\
6 & 1.020 & $1.459 \times 10^{4}$ & 0.012 & 0.185 & $3.090 \times 10^{3}$ & 0.316 \\
7 & 0.522 & $1.056 \times 10^{4}$ & 0.016 & 0.017 & $1.305 \times 10^{3}$ & 0.501 \\
\hline
\end{tabular}

